c) L'indice de Wauters-Polenske est souvent très élevé ; ensuite, da maladie influence le taux en l'exagérant des acides volatils insolubles.

d) L'indice de saponification montre quelque abaissement d'accord avec l'abaissement de l'indice de R. M., mais il reste en tous cas dans les limites normales.

e) L'indice d'iode (de Нӥвц) a été trouvé dans deux de nos trois cas remarquablement élevé.

f) La solubilité de la matière grasse du lait dans l'acide acétique diminue, ce que montre l'indice de Savini très élevé.

Dans la pratique, il peut arriver que l'on analyse le beurre venant de vaches atteintes de la fièvre aphteuse. En se fiant à l'indice de R. M, et à celui de W. P., ce beurre pourrait être arrêté comme falsifié par l'addition de la graisse de coco. Mais il diffère, par ailleurs, du beurre ainsi falsifié par l'indice de réfraction, par l'indice d'iode et par celui de Savinı. Ces indices sont pour la matière grasse de coco toujours beaucoup plus bas que pour la matière grasse du beurre. Dans ce cas, j'attache beaucoup d'importance à l'indice de SAvinI qui décide tout simplement du cas, car au lieu de s'abaisser, il s'élève notablement.

L'étude plus haut citée de l'influence de la fièvre aphteuse sur la formation de la matière grasse du lait a été faite sur un petit nombre de cas ; et, aussi ne saurait-elle être définitive. Le devoir de chercheurs que la question intéresserait est de pousser l'analyse dans plus de détails encore.

\title{
SUR LA VALEUR RELATIVE DE L'EPREUVE DE LA REDUCTASE DANS LE CONTROLE HYGIÉNIQUE DU LAIT,
}

\author{
par M. le $\mathrm{D}^{\mathrm{r}}$ Alberto de PAUlaA RODRigUES,
}

Chef du Service du Contrôle du Lait et des Produits Laitiers du Département de la Santé Publique au Brésil (Rio de Janeiro).

Le pouvoir réducteur du lait de vache s'exerce sur le sonfre ou sur certaines matières colorantes dérivées de la houille, dont le bleu de méthylène est la plus généralement employée.

L'action réductrice, soit en changeant le soufre en acide sulihydrique, soit en transformant le bleu de méthylène en leuco-dérivé, 
s'exerce fait par la fixation de deux atomes de $\mathrm{H}$ (sur un corps. simple : $\mathrm{S}+\mathrm{H}^{2}=\mathrm{H}^{2} \mathrm{~S}$ ) ou sur un corps complexe.

$$
\mathrm{C}^{46} \mathrm{II}^{18} \mathrm{~N}^{3} \mathrm{SCl}+\mathrm{H}^{2}=\mathrm{C}^{16} \mathrm{II}^{20} \mathrm{~N}^{3} \mathrm{SCl}
$$

(Bleu de mèthylène en nature) $=$ (Leuco-dérivé du bleu de mèthylène)

HefFerer n'a pas de raison pour donner un nom particulier : hydrogénase, au ferment réducteur du corps simple. Egalement nous ne devons pas admettre la désignation de Aldéhydocatalase (de SMIDT) ou Aldéhydoréductase (de O. Jensen) pour la réduction opérée en présence du formaldéhyde. L'action du formol dans le réactif de SchARDINGER n'est plus qu'une action accélératrice particulière à cet. aldéhyde à une certaine dose et qu'on constate sur d'autres ferments du lait.

C'est la propriété réductrice du lait sur le bleu de méthylène qui a été utilisée dans son contrôle hygiénique, les expérimentateurs ayant observé la corrélation étroite qui existe entre la contamination microbienne du lait et la rapidité avec laquelle il décolore une solution de bleu de méthylène.

Parmi ces observateurs, Barthel, le premier, a affirmé que la décoloration du bleu par le lait se fait exclusivement par l'action des microbes (1911 - Die Methoden zur Untersuchung oon Milch Traduction allemande de l'original suédois).

Ultérieurement l'auteur suédois se contredit (Skandinawisk Veterinartidsskift, 1916) et affirme que le lait de vache a des propriétés réductrices caractéristiques indépendantes de la contamination bactérienne.

Eichwald (Probleme und Aufgaben der Nahrungsmittelchemie) a. raison quand il dit qu'on ne doit pas attendre de grand̄s résultats de l'épreuve de la réductase, source d'erreurs découlant des changeantes particularités des bactéries variées qui se développent dans le lait. C'est justement ce que nous avons vérifiépendant trois années d'observations dans le contrôle hygiénique du lait à Rio de Janeiro. Si nous mettons en comparaison l'épreuve de la réductase avec celle de l'ensemencement du lait dans des boites de PETri, avec gélose peptone (1) pendant 72 heures à $37^{\circ} \mathrm{C}$, conditions les meilleures pour obtenir la germination de la plus grande partie des microbes, qui peuvent se trouver dans un échantillon donné, nous vérifierons la discordance des résultats obtenus avec les étalons admis par Orla Jensen et adoptés dans la dernière édition (1923) des Standard Methods of Milk Analysis aux Etats-Unis (2).

(1) Gélose : 20, Peptone : 10, Extrait de viande . 5, NaGl : 5, Eau : 100. Neutraliserle milieu avec une solution $\mathrm{N} / 10$ de soude.

(2) Le Lait publiera en 1925 la traduction française de ces Standards (R.). 
Etalons d'Orla Jensen, admis par les Standard Methods :

A. bons laits. - Décoloration du bleu après 5 heures et demi. Quantité de microbes : 500.000 par cc.

B. laits réguliers. - Décoloration du bleu de 5 heures et demi à 2 heures. - Quantité de microbes : de 500.000 à 4 millions par centimètre cubique.

C mauvais laits. - Décoloration du bleu de 2 heures à 20 minutes. Quantité de microbes : de 4 à 20 millions par centimètre cubique.

D. laits très mauvais. - Décoloration du bleu en 20 minutes ou moins encore; avec plus de 20 millions de microbes par centimètre cubique.

\section{Observations du contrôle hygiénique du lait à Rio de Janeiro.}

EpreuveM.: Solution simple de bleu de méthylène.

4 heures.

2 -

Pas de décoloration. 4 heures.

4

Après 5 heures.

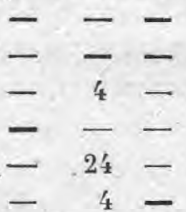

Pas de décoloration.

Après 4 heures.

$-5 \quad-$
Dans $4 \quad-1 / 2$.
Epreuve M. F.: Solution de Schardinger

(1)

45 minutes.

2 heures.

Pas de décoloration. 30 minutes.

$\begin{array}{ll}- & - \\ - & - \\ - & - \\ - & - \\ 40 & - \\ - & - \\ 35 & - \\ 42 & - \\ 40 & - \\ 75 & - \\ - & - \\ 25 & - \\ - & -\end{array}$

Degrés d'acidité Dornic.

Pourcentage de microbes par centimètre cubique révélés dans les cultures de gélose-peptone.

$18^{\circ} \mathrm{D}$.

5.000 .000

$19^{\circ} \mathrm{D}$.

21.500 .000

$20^{\circ} \mathrm{D}$.

16.000000

$19^{\circ} \mathrm{D}$.

18.000.000

$20^{\circ} \mathrm{D}$.

15.000 .000

$19^{\circ} \mathrm{D}$.

2.000 .000

$170 \mathrm{D}$.

1.500 .000

$18^{\circ} \mathrm{D}$.

2.000 .000

$20^{\circ} \mathrm{D}$.

10.000 .000

$19^{\circ} \mathrm{D}$.

5.000 .000

$21^{\circ} \mathrm{D}$.

10.500 .000

$19^{\circ} \mathrm{D}$.

1. 400.000

$19^{\circ} \mathrm{D}$.

20.000 .000

$20^{\circ} \mathrm{D}$.

$18^{\circ} \mathrm{D}$.

10.000 .000

$18^{\circ} \mathrm{D}$.

5.000 .000

$20^{\circ}$ D.
30.000 .000

25.000 .000

La solution et la technique ulilisées par nous sont celles de Gerber et Ottiker, en Suisse ; Barthel, en Suède ; Morres, en Allemegne ; Kufferath, ea Belgique et Oria Jensen, au Danemark.

(1) Solution alcoolique saturée de bleu de méthylène et formol à $40 \%$ : âà 5 cent, cub, ; Eau distillée: 190 cent. cubiques. Température de $55^{\circ}$ a $50^{\circ}$ C. (Uptimum avec accélérateur) 
Les Américains du nord, dans leurs expériences, affirment (Standard Methods) qu il y a des bactéries présentes dans le lait qui ne se révèlent pas à la culture sur les plaques de gélose et cependant peuvent décolorer le bleu, c'est-à-dire, yue l'épreuve de la réductase,dans le contrôle du lait, aurait plus de signification. Je ne pense pas de la sorte; ce sont les propriétés réductrices, inhérentes au lait, surajoutées au pouvoir réducteur de certains microbes, qui mènent à cette erreur d'appréciation des résultats obtenus par les Américains du nord.

D'autre part, c'est un fait susceptible d'observation courante que les bactéries du lait agissent, dans la décol ratiou du leu le méthylène ajouté, en épuisant l'oxygène dissous, c'est-à-dire indirectement. Tant qu'il y a de l'oxygène dans le lait, la décoloration ne se fait pas. La couche superficielle du lait en contact avec l'air ne se décolore pas. Pour l'épreuve de la réductase, il faut maintenir l'échantillon du lait dans des tubes spéciaux fermés hermétiquement, ou dans des tubes à essais si l'on isole le lait de l'air, avec une couche de vaseline liquide. Burrt et Kürsteiner ont démontré que, dans un lait fraîchement stérilisé, on peut obtenir la décoloration du bleu simplement en empêchant l'absorption de l'oxygène.

Nous avons observé dans les tubes à essais avec du lait et utilisés dans l'épreuve de la réductase, maintenus à l'abri de l'air et abandonnés à la fermentation spontanée, après 48 heures (à la température ambiante de $20^{\circ}$ C.), la recoloration du bleu toutes les fois qu'il y avait production de gaz pendant la coagulation du lait. Certainement ce sont des microbes anaérobies qui produisent de l'oxygène, dont la réapparition dans le lait provoque l'oxydation du leuco-dérivé en bleu de méthylène.

De ces observations déeoulent un autre fait: plus un lait se trouve aéré par effet de la traite, manipulation et transport, plus lentement le bleu sera décoloré.

Erchwald affirme qu'on doit mettre en évidence le phénomène de l'adsorption de la matière colorante, particulière aux collö̈des. Dans le lait, nous trouvons dans cet état de dispersion de la matière, la caséine et le phosphate de chaux, formant un complexe extrêmement solide, mais d ordre physique.Nous pouvons considérer ce colloïde du lait comme un émulsoïde qui, sous l'influence de certains facteurs, tels que l'acide laetique, passe à l'état de $g e l$; c'est-à-dire que sous l'action des ferments lactiques, le complexe colloïdal caséine-phosphate de chaux est floculé sous l'aspect particulier d'un tissu de mailles : c'est le phénomène de la coagulation.

Pourquoi n'admettons-nous ici dans la production de la coagulation que les ferments lactiques? C'est que, dans toutes nos observations, 
les laits étaient riches en acide lactique (Voyez les degrés d'acidité constatés dans le tableau ci-dessus : toujours au-dessus de $15^{\circ}$ Dornic.

C'est que dans ces laits prédominait la flore acidifiante coagulante, figurée par le Bacterium lactis acidi, le Streptococcus lactis acidi et le Bacillus Bulgaricus ; e'étaient des laits acides, en commencement de fermentation acide pure. A Rio de Janeiro, et certainement dans tous les climats où la température moyenne de l'année est toujours au-dessus de $15^{\circ}$ centigrades, il est impossible de trouver des laits sans acide lactique, résultant de l'action des microbes acidifiants sur la lactose. On sait que le Bacterium lactis acidi commence à pous ser avec intensité à partir de $15^{\circ} \mathrm{C}$. où mème dans quelques variétés, à partir de $10^{\circ} \mathrm{C}$. Son optimum de température oscille entre $30^{\circ}$. à $35^{\circ}$.

A Rio de Janeiro, silué un demi degré au-dessus du tropique du Capricorne, la température moyenne de l'année est $20^{\circ} \mathrm{C}$. ; à la saison chaude, $30^{\circ} \mathrm{C}$. avec maximum de $35^{\circ} \mathrm{C}$. ; à la saisen fraîche (3 mois) minimum de $15^{\circ}, 5$ et $10^{\circ} \mathrm{C}$. exceptionnellement.

C'est pour cela qne nos observations justifient l'affirmation d'Orla JENSEN ; l'épreuve de la réductase dans le lait n'a de valeur que quand il n'y a pas encore d'acide lactique produit, hypothèse impossible dans les pays chauds, mais naturellement soutenable dans les climats. semblables à celui du Danemark.

Au contraire, nos observations contestent l'affirmation des auteurs des Standard Methods du Nord-Amérique : à savoir que les bactéries lactiques ordinaires ont un pouvoir réducteur apparemment plus marqué sur le bleu de méthylène, sanf dans le cas où ces bactéries lactiques, aux Etats-Unis, différeraient de celles obtenues à Rio de Janeiro (Bact. lactis acidi, Streptococcus, lactis acidi ou chez nous importées, tel le Bacillus Bulgaricus). Nous sommes portés à le penser, parce que des cultures pures de ces bactéries dans du lait écrémé, mises en présence de la solution de bleu de méthylène dans la. proportion prescrite par les Standard Methods, ne font pas la réduction : le milieu reste coloré et la culture deviendra même stérilisée.

Nous sommes amenés vers l'hypothèse plus rationnelle: que le trouble apporté au complexe colloïdal du lait, du fait de la présence de l'acide lactique, résulte de la fermentation acide pure causée par des microbes acidifiants, germinant à la température favorable.

A Rio de Janeiro ou aux Etats-Unis, partout, le lait a cette a harmonie », dont parle le Professeur Porchen, par rapportà son origine, destination et composition. Cette “harmonie » est troublée par la présence de l'acide lactique, étranger à la composition normale du lait, puisque le seul acide organique trouvé et tout à fait caractéristique du lait, c'est l'acide eitrique. 